\title{
Molecular preservation of the pigment melanin in fossil melanosomes
}

Johan Lindgren ${ }^{1}$, Per Uvdal ${ }^{2,3}$, Peter Sjövall ${ }^{4}$, Dan E. Nilsson ${ }^{5}$, Anders Engdahl$^{2}$, Bo Pagh Schultz ${ }^{6}$ \& Volker Thiel ${ }^{7}$

Fossil feathers, hairs and eyes are regularly preserved as carbonized traces comprised of masses of micrometre-sized bodies that are spherical, oblate or elongate in shape. For a long time, these minute structures were regarded as the remains of biofilms of keratinophilic bacteria, but recently they have been reinterpreted as melanosomes; that is, colour-bearing organelles. Resolving this fundamental difference in interpretation is crucial: if endogenous then the fossil microbodies would represent a significant advancement in the fields of palaeontology and evolutionary biology given, for example, the possibility to reconstruct integumentary colours and plumage colour patterns. It has previously been shown that certain trace elements occur in fossils as organometallic compounds, and hence may be used as biomarkers for melanin pigments. Here we expand this knowledge by demonstrating the presence of molecularly preserved melanin in intimate association with melanosome-like microbodies isolated from an argentinoid fish eye from the early Eocene of Denmark.

\footnotetext{
${ }^{1}$ Department of Geology, Lund University, 22362 Lund, Sweden. ${ }^{2}$ MAX-IV laboratory, Lund University, 22100 Lund, Sweden. ${ }^{3}$ Chemical Physics, Department of Chemistry, Lund University, 22100 Lund, Sweden. ${ }^{4}$ SP Technical Research Institute of Sweden, Chemistry and Materials, 50115 Borås, Sweden. ${ }^{5}$ Department of Biology, Lund University, 22362 Lund, Sweden. ${ }^{6}$ Museum Salling - Fur Museum, 7884 Fur, Denmark. ${ }^{7}$ Geobiology Group, Geoscience Centre, University of Göttingen, 37077 Göttingen, Germany. Correspondence and requests for materials should be addressed to

J.L. (email: johan.lindgren@geol.lu.se).
} 
$\mathrm{n}$ vertebrate animals, melanins are complex, polymeric pigments located in a variety of tissues, including the skin, hair, feathers and eyes, where they serve a number of important biological functions, such as protection against short-wavelength light ${ }^{1,2}$. Additionally, they provide the integument and its appendages with the majority of black, grey, brown and reddish tones, thereby contributing to camouflage, thermoregulation and social signalling ${ }^{3}$. Melanins are synthesized and packaged in melanosomes; that is, lysosome-related organelles generated by melanocytes and pigment-epithelial cells ${ }^{4}$. Melanosomes are typically between 500 and $2000 \mathrm{~nm}$ in the long axis direction and they occur in a broad array of shapes, ranging from sub-spherical to distinctly ellipsoi$\mathrm{dal}^{4}$. Whereas melanin granules are stored permanently in eye pigment cells, mature dermal melanosomes migrate from melanocytes to neighbouring keratinocytes (keratin-producing cells) for ultimate incorporation into developing feathers and hairs to create colour patterning 3,4

Putative fossil melanosomes have been described from feathers, hairs and vertebrate eyes dating to the Jurassic, and are preserved primarily as carbonaceous traces ${ }^{5-17}$, although replication by authigenic minerals also has been reported ${ }^{7,18}$. If indeed being the remains of ancient pigment organelles, then these microbodies would greatly improve our understanding of, for example, the evolutionary origin, function and colouration of feathers in extinct animals ${ }^{8-11,13-17}$. However, because both the shape and size of melanosomes correspond well with those of bacteria ${ }^{8,10,13}$, it is necessary to distinguish the two. Previous claims of melanosomes in multimillion-year-old animals have been based chiefly on structure $^{8-11,16,17}$, although some trace elements that are present in fossil soft tissues rich in melanosome-like microbodies recently have been suggested to possess melanin affinity ${ }^{15}$. Nonetheless, because microorganisms are capable of adsorbing and concentrating trace metals $^{19}$, a deeper understanding of the chemistry of the proposed fossil melanosomes is needed to avoid controversies and plausible alternative interpretations ${ }^{13,20}$.

Here we demonstrate that the organic content of melanosomelike microbodies isolated from a fossil fish eye is dominated by the pigment melanin. This result was obtained by time-of-flight secondary ion mass spectrometry (ToF-SIMS) - a powerful technique capable of simultaneous identification and mapping of molecules and chemical structures in biological samples at high spatial resolution ${ }^{21}$-combined with infrared (IR) microspectroscopy, transmission electron microscopy (TEM) and scanning electron microscopy (SEM).

\section{Results}

Identification of microbodies in a fossil fish eye. The material examined, a virtually complete argentinoid fish (Museum SallingFur Museum; FUM-N-2050), was collected from the early Eocene $(\sim 54 \mathrm{Ma})$ Fur Formation of Denmark ${ }^{22,23}$, without application of preservatives (see Supplementary Methods online). The specimen is flattened but otherwise well preserved, comprising an almost complete and articulated skeleton with a distinct brownish pigmentation located firmly within the orbit (Fig. 1a,b). Under SEM, the dark
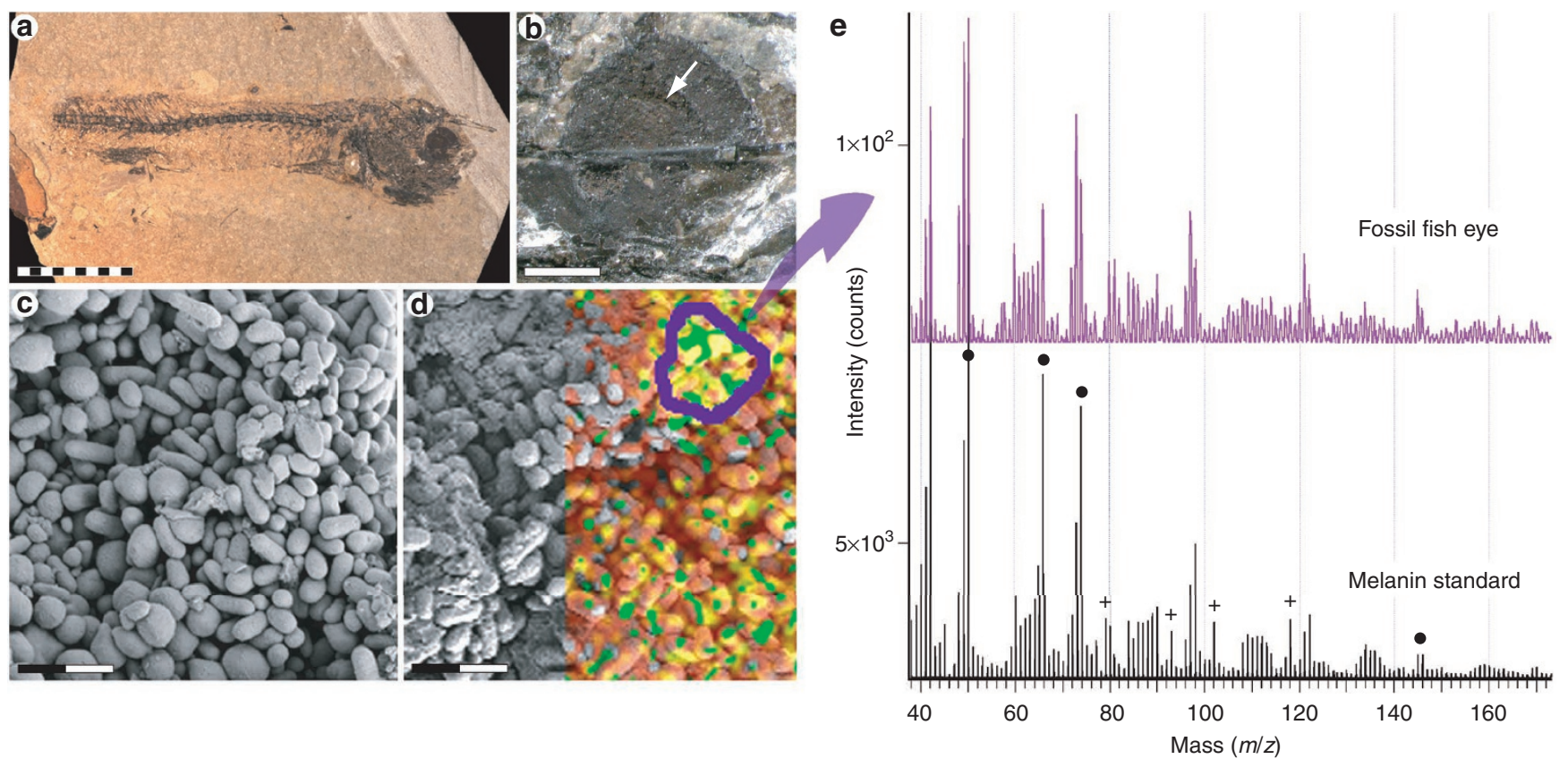

Figure 1 | Negative ion ToF-SIMS spectra of melanin and microbodies from FUM-N-2050. (a) Optical photograph of the specimen. Scale bar: $10 \mathrm{~mm}$. (b) Close-up of brownish matter located in the orbit; position of the area analysed by SEM, TEM, ToF-SIMS and IR microspectroscopy indicated by an arrow. Scale bar: $1 \mathrm{~mm}$. (c) Detail of the eye (SEM image) showing closely spaced, elongate and oblate melanosome-like structures preserved as solid bodies. Scale bar: $2 \mu \mathrm{m}$. (d) A semi-transparent ion image showing the distribution of melanin-derived ions (identified from natural and synthetic melanin standard spectra; Table 1; Supplementary Fig. S3) superimposed onto a SEM image of tightly packed melanosome-like bodies. The added signal intensity from $\mathrm{C}_{3} \mathrm{~N}^{-}(\mathrm{m} / \mathrm{z} 50), \mathrm{C}_{3} \mathrm{NO}^{-}\left(\mathrm{m} / \mathrm{z}\right.$ 66) and $\mathrm{C}_{5} \mathrm{~N}^{-}(\mathrm{m} / \mathrm{z}$ 74) is shown in orange-yellow, whereas areas high in signal from peaks at $\mathrm{m} / z 145$ and $\mathrm{m} / z 146$ are shown in green (see Table 1 for assignments). The purple line demarcates the area from which the mass spectrum presented in (e) ('fossil fish eye') was collected. Scale bar: $2 \mu \mathrm{m}$. (e) Negative ToF-SIMS spectra representing the eye of FUM-N-2050 and a natural melanin standard (from Sepia officinalis). The fossil fish eye spectrum was reconstructed from a $4 \mu \mathrm{m}^{2}$ area in a measurement at $50 \times 50 \mu \mathrm{m}^{2}$ acquired at high spatial resolution (mass resolution $\mathrm{m} / \Delta m \sim 300$ ) to allow for analysis of ToF-SIMS data specifically from within the selected area marked in (d), whereas the melanin standard spectrum was recorded at high mass resolution $(m / \Delta m ~ 5000)$. The close agreement between the two spectra (both with regard to their peak positions and relative intensity distributions) provides compelling evidence for a high melanin content in the eye of FUM-N-2050. Filled circles indicate peaks used to produce the ion image in (d), whereas crosses indicate peaks from inorganic ions that are not part of the melanin structure (and not present in the synthetic melanin standard spectrum; Supplementary Fig. S3). 
a

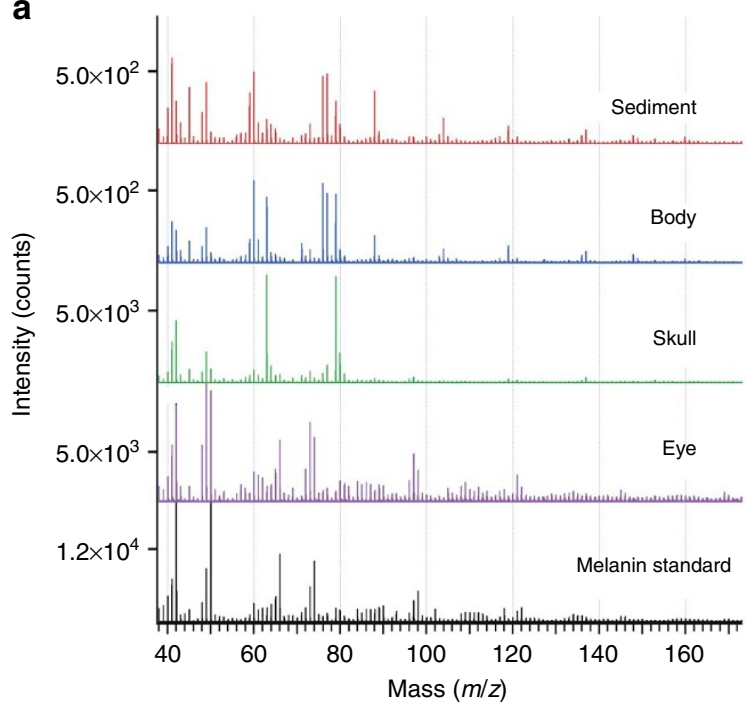

b

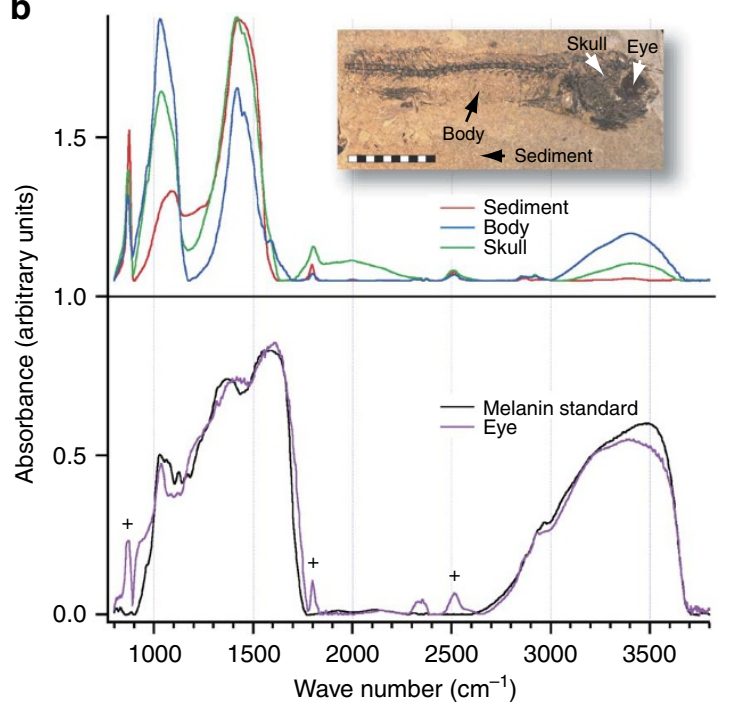

Figure 2 | Negative ion ToF-SIMS spectra and IR absorbance spectra of melanin and parts of FUM-N-2050. (a) Negative ion ToF-SIMS spectra obtained from a natural melanin standard sample and different parts of FUM-N-2050 (as indicated by arrows in the inset photograph in (b). Scale bar: $10 \mathrm{~mm}$ ). All spectra were recorded at high mass resolution. Only the fossil fish eye spectrum shows strong similarities with the melanin standard spectrum; all other spectra are dominated by peaks from inorganic ions, such as $\mathrm{PO}_{2}{ }^{-}$at $\mathrm{m} / \mathrm{z} 63, \mathrm{PO}_{3}{ }^{-}$at $\mathrm{m} / \mathrm{z} 79$ and $\mathrm{Si}_{x} \mathrm{O}_{\mathrm{y}}{ }^{-}$at $\mathrm{m} / \mathrm{z} 60,76,77$ and 88 . (b) IR absorbance spectra of various structures of FUM-N-2050 and a natural melanin standard. The spectrum from the eye exhibits broad-band absorbance in the $900-1750$ and $2700-3700 \mathrm{~cm}^{-1}$ regions, consistent with that of the melanin standard. The low-energy region is characteristically broad and featureless, and the vibrational modes are typically delocalized collective ring motions without any specific normal mode character. Crosses represent contributions from calcite ${ }^{35}$; note that these lines are also present in the spectra from the sediment, body and skull. The infrared line at $874 \mathrm{~cm}^{-1}$ is assigned to the out-plane bend mode of $\mathrm{CO}_{3}$, whereas the remaining two lines (at 1800 and $2517 \mathrm{~cm}^{-1}$ ) are assigned to an overtone and a binary combination mode, respectively. In contrast to the eye spectrum, the three infrared spectra obtained from the skull, body and sediment are clearly different from that of the natural melanin standard. In addition to the above-mentioned lines associated with calcite, there is a broad peak at $\sim 1415 \mathrm{~cm}^{-1}$ (particularly in the sediment) that contains some contributions from the asymmetric stretch mode of the carbonate in calcite. The peak at $1000-1100 \mathrm{~cm}^{-1}$ in the skull and body spectra could be associated with, for example, calcium phosphate. For the sediment spectrum, the absence of OH stretches in the $3000 \mathrm{~cm}^{-1}$ region and the shape of the $1000-1100 \mathrm{~cm}^{-1}$ peak are consistent with quartz.

matter showed masses of sub-spherical, oblate and prolate bodies, about 400-1500 nm long (Fig. 1c). Additionally, elemental mapping using EDX (energy-dispersive X-ray spectrometry) revealed that the eye contains more carbon (and slightly more sulphur) than do the surrounding bony tissues and sediments (Supplementary Fig. S1). Microbodies studied under TEM revealed a largely homogenous internal content consisting of dense matter (Supplementary Fig. S2).

Localization of melanin to the fossil fish eye. To analyse the molecular content of the melanosome-like bodies, samples of grains were removed from the eye (as well as from other parts of the fossil and surrounding sediments) using a sterile scalpel, and subjected to ToF-SIMS analysis. The resulting mass spectra, which are formed by secondary ions emitted from the sample surface when a focussed beam of high energy ions is scanned over it, provide spatially resolved molecular information and a detailed chemical fingerprint of the sample surface. A close agreement was observed between spectra obtained from the eye of FUM-N-2050 and that of a natural melanin standard, both with regard to the positions of the observed peaks and their intensity distributions (Fig. 1e; Supplementary Fig. S3). The striking spectral agreement between the fossil fish eye and the melanin standard strongly suggests that the former contains high concentrations of melanin. Furthermore, the signal intensity in terms of number of detected secondary ions per incident primary ion (that is, secondary ion (SI) yield) in the fish eye spectrum (Supplementary Table S1) is comparable to that of the melanin standard spectrum (the low counts in the former spectrum is a consequence of it representing only a small portion of the total analysis area, see caption to Fig. 1e), which is also consistent with melanin being a major molecular constituent of the analysed surface.
The spectrum from the eye of FUM-N-2050 presented in Fig. 1e was obtained from within the $\sim 4 \mu \mathrm{m}^{2}$ area enclosed by the purple line in Fig. 1d (which, according to a SEM image of the same area, contains almost exclusively melanosome-like microbodies). Moreover, an ion image showing the spatial signal intensity distribution of characteristic melanin peaks (selected from the melanin standard spectrum; Fig. 1e) superimposed onto the SEM image (Fig. 1d), clearly demonstrates that the signal intensity from the characteristic melanin peaks spatially correlates with the melanosome-like structures (see also Supplementary Figs S4, S5). ToF-SIMS spectra from other parts of FUM-N-2050, including the skull, body and surrounding sediments, were distinctively different from those obtained from the microbody-rich eye (Fig. 2a), to suggest dissimilar chemical compositions of primarily inorganic components, such as phosphates (skull and body) and silicates (body and sediments).

Although the data obtained at high spatial resolution demonstrate that the melanin-like spectra originate from the melanosomelike microbodies (Fig. 1d,e), high mass resolution spectra from the fossil fish eye showed peak positions virtually indistinguishable from those of the melanin standard (Table 1; Supplementary Fig. S3), thereby corroborating their assignments to melanin. Furthermore, all major peaks in the high mass resolution spectra from the natural melanin standard and fossil fish eye (as well as from a synthetic melanin standard; Supplementary Fig. S3) can be tentatively assigned to fragment ions that are consistent with the chemical structure of nitrogen-rich melanins (Fig. 3, Table 1$)^{24}$.

These observations were corroborated by IR microspectroscopy consistent with previous infrared studies of fossil melanin ${ }^{14,15}$. The vibrational spectrum for the fossil microbodies is presented along with a spectrum for a natural melanin standard and other tissues 
Table 1 | Tentative assignments and $\mathrm{m} / \mathrm{z}$ values for peaks in negative ToF-SIMS spectra from melanin and fossil microbodies.

\begin{tabular}{|c|c|c|c|c|}
\hline Tentative assignment & Theoretical mass & $\begin{array}{c}\text { Synthetic melanin } \\
\text { standard }\end{array}$ & $\begin{array}{l}\text { Sepia melanin } \\
\text { standard }\end{array}$ & $\begin{array}{c}\begin{array}{c}\text { Fossil sample } \\
\text { (FUM-N-2050) }\end{array} \\
\end{array}$ \\
\hline $\mathrm{C}_{2}$ & 24.000 & 23.999 & 24.000 & 24.000 \\
\hline $\mathrm{C}_{2} \mathrm{H}$ & 25.008 & 25.009 & 25.008 & 25.009 \\
\hline $\mathrm{CN}$ & 26.003 & 26.006 & 26.004 & 26.006 \\
\hline$C_{3}$ & 36.000 & 36.000 & 36.000 & 36.000 \\
\hline $\mathrm{C}_{3} \mathrm{H}$ & 37.008 & 37.009 & 37.008 & 37.009 \\
\hline $\mathrm{C}_{2} \mathrm{~N} / \mathrm{C}_{3} \mathrm{H}_{2}$ & $38.003 / 38.016$ & $38.003 / 38.014$ & $38.004 / 38.016$ & $38.002 / 38.015$ \\
\hline $\mathrm{C}_{2} \mathrm{HO}$ & 41.003 & 41.006 & 41.007 & 41.006 \\
\hline CNO & 41.998 & 42.002 & 42.000 & 42.000 \\
\hline $\mathrm{C}_{4}$ & 48.000 & 48.000 & 48.001 & 48.002 \\
\hline $\mathrm{C}_{4} \mathrm{H}$ & 49.008 & 49.010 & 49.010 & 49.011 \\
\hline $\mathrm{C}_{3} \mathrm{~N}$ & 50.003 & 50.007 & 50.005 & 50.007 \\
\hline $\mathrm{C}_{5}$ & 60.000 & 60.002 & 60.002 & 60.001 \\
\hline $\mathrm{C}_{4} \mathrm{HO} / \mathrm{H}_{3} \mathrm{NO}_{3}$ & $65.003 / 65.012$ & 65.009 & 65.013 & 65.010 \\
\hline $\mathrm{C}_{3} \mathrm{NO}$ & 65.998 & 66.003 & 66.001 & 66.003 \\
\hline $\mathrm{C}_{6}$ & 72.000 & 72.001 & 72.003 & 72.001 \\
\hline $\mathrm{C}_{6} \mathrm{H} / \mathrm{C}_{2} \mathrm{H}_{3} \mathrm{NO}_{2}$ & $73.008 / 73.016$ & 73.010 & 73.010 & 73.012 \\
\hline $\mathrm{C}_{5} \mathrm{~N} / \mathrm{C}_{2} \mathrm{H}_{2} \mathrm{O}_{3}$ & $74.003 / 74.001$ & 74.006 & 74.006 & 74.008 \\
\hline $\mathrm{C}_{7}$ & 84.000 & 84.000 & 84.002 & 84.002 \\
\hline $\mathrm{C}_{7} \mathrm{H}$ & 85.008 & 85.008 & 85.010 & 85.008 \\
\hline $\mathrm{C}_{6} \mathrm{~N} / \mathrm{C}_{7} \mathrm{H}_{2}$ & $86.003 / 86.015$ & 86.009 & 86.009 & 86.012 \\
\hline $\mathrm{C}_{6} \mathrm{HN}$ & 87.011 & 87.015 & 87.014 & 87.016 \\
\hline $\mathrm{C}_{6} \mathrm{H}_{2} \mathrm{~N} / \mathrm{C}_{3} \mathrm{H}_{4} \mathrm{O}_{3}$ & $88.018 / 88.016$ & 88.015 & 88.014 & 88.016 \\
\hline $\mathrm{C}_{6} \mathrm{HO} / \mathrm{C}_{2} \mathrm{H}_{3} \mathrm{NO}_{3}$ & $89.002 / 89.011$ & 89.012 & 89.015 & 89.013 \\
\hline $\mathrm{C}_{5} \mathrm{NO}$ & 89.998 & 90.003 & 90.002 & 90.003 \\
\hline $\mathrm{C}_{8}$ & 96.000 & 95.999 & 96.002 & 96.001 \\
\hline$C_{10}$ & 120.000 & 120.000 & 119.996 & 119.999 \\
\hline $\mathrm{C}_{10} \mathrm{H} / \mathrm{C}_{6} \mathrm{H}_{3} \mathrm{NO}_{2}$ & $121.008 / 21.016$ & 121.008 & 121.010 & 121.012 \\
\hline $\mathrm{C}_{9} \mathrm{~N}$ & 122.004 & 122.005 & 122.006 & 122.008 \\
\hline $\mathrm{C}_{12} \mathrm{H} / \mathrm{C}_{8} \mathrm{H}_{3} \mathrm{NO}_{2}$ & $145.008 / 145.016$ & 145.006 & 145.009 & 145.008 \\
\hline $\mathrm{C}_{11} \mathrm{~N}$ & 146.003 & 146.006 & 146.007 & 146.007 \\
\hline
\end{tabular}

and sediments of FUM-N-2050 in Fig. 2b. There are clear and significant similarities between the two spectra representing the fossil microbodies and the melanin standard, to suggest a similar molecular structure and composition ${ }^{25}$. In contrast, the spectra obtained from the skull, body and sediments are considerably different from the melanin standard, and instead they are consistent with calcite, apatite and quartz; that is, minerals expected to be found in these parts of the specimen (Fig. 2b).

\section{Discussion}

The molecular analyses performed in this study demonstrate that the chemical composition of the fossil microbodies variably assigned to as bacterial biofilms $s^{5,6,12}$ or melanosomes ${ }^{8-11,14-17}$, at least in FUM-N-2050, is dominated by melanin or a molecular structure very similar to this pigment. Although microorganisms can synthesize melanins ${ }^{26}$, we argue against the possibility that the melanin-containing structures represent replacement bacteria because the pigment is highly concentrated and localized specifically to melanosome-like bodies in a tissue where melanin must have been a major constituent in life (cf. retinal melanosomes in an extant three-spined stickleback fish eye; Fig. 4). Additionally, it has previously been shown that melanosome-like bodies only occur in dark bands of fossil-banded feathers ${ }^{8}$, and that their organization and layering ultrastructure are identical to those of coloured feathers in extant birds ${ }^{9}$. Furthermore, it was recently demonstrated that certain trace elements, such as copper and zinc, are present in both ancient and modern melanosome-bearing tissues, and hence may act as biomarkers for melanin-derived compounds in fossils ${ }^{15}$. Finally, our ToF-SIMS analyses of various types of modern microbial mats (Supplementary Figs S6, S7) revealed chemical 

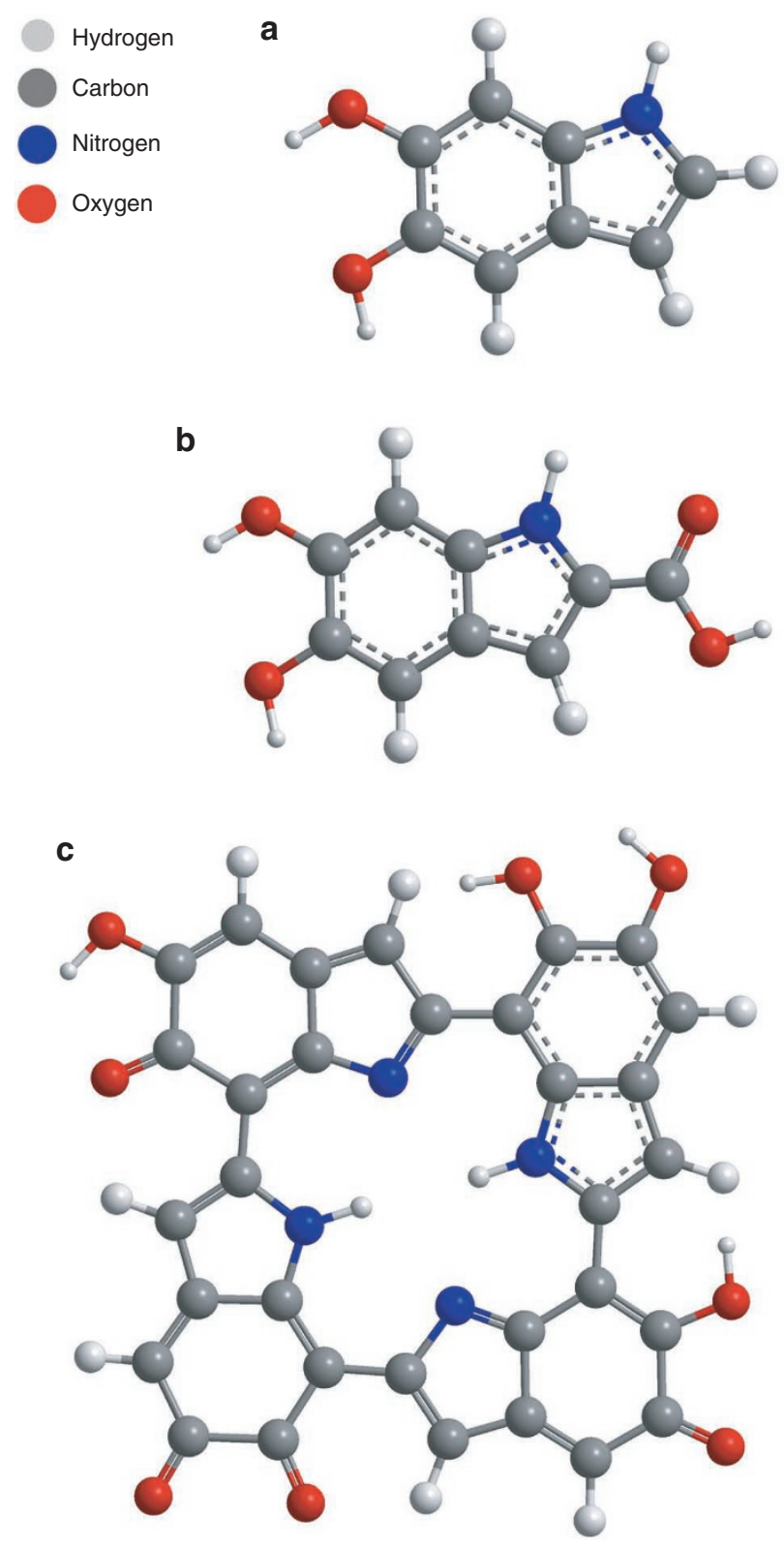

Figure 3 | Basic monomeric units and oligomeric structure of melanin. Monomeric units of melanin; (a) 5,6-dihydroxyindole (DHI) and (b) 5,6dihydroxyindole-2-carboxylic acid (DHICA) (modified from ref. 33). (c) Proposed oligomeric structure model of melanin (modified from ref. 36).

signatures that were significantly different from those of melanins, something that was particularly obvious in high-resolution mass spectra (Supplementary Fig. S7). The molecular specificity of the ToF-SIMS and IR microspectroscopic results was also tested by acquiring spectra of hemin chloride and chlorophyll $a$-two porphyrin-related compounds with molecular structures similar to that of melanin (Supplementary Fig. S8) - and comparing these spectra to those of the fossil fish eye and melanin standards. These compounds were chosen because chlorophylls and cytochromes (that is, heme derivates) are generally considered as the main biological precursors of so-called 'fossil porphyrins' (or 'geoporphyrins'), which are common in sediments containing organic matter ${ }^{27,28}$. Although the resulting mass spectra from melanin, hemin chloride and chlorophyll $a$ have several peaks in common, their intensity distributions were distinctly different from one another (Supple-

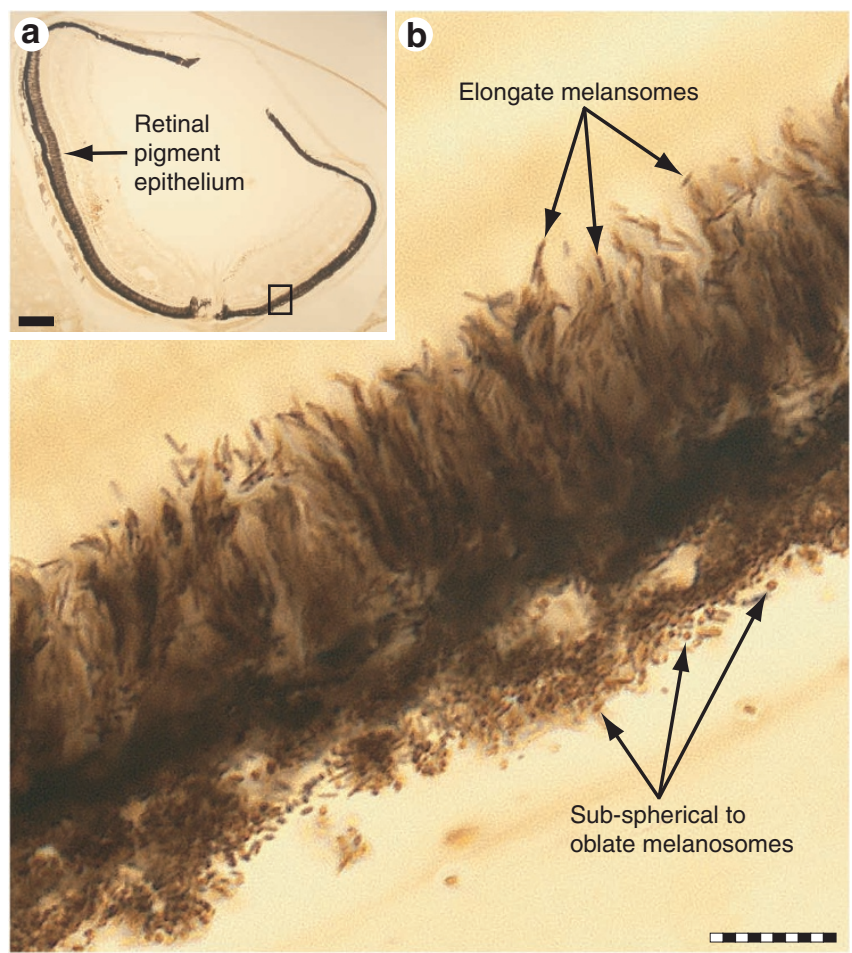

Figure 4 | Retinal melanosomes in an extant fish eye. (a) Cryosection through an eyecup of a juvenile three-spined stickleback fish (Gasterosteus aculeatus) where the retinal pigment epithelium (RPE) occurs as dark, brownish tissue (mirror image of original photograph). The rectangle indicates the area shown in (b). Scale bar: $100 \mu \mathrm{m}$. (b) Close-up of the RPE in G. aculeatus. Note that elongate melanosomes primarily occur in the distal parts of the RPE, whereas the proximal parts chiefly contain sub-spherical to oblate melanosomes. Degradation and ultimate collapse of the RPE during the process of fossilization would result in a mixture of melanosome morphologies located on top of each other, as in the eye of FUM-N-2050. Scale bar: $10 \mu \mathrm{m}$.

mentary Fig. S9). Importantly, whereas the two porphyrin-related compounds showed a number of peaks with very similar intensity distributions (which are probably related to the ring-structure of porphyrins), a close spectral agreement in the entire mass range up to $\mathrm{m} / \mathrm{z} 160$ was found only between the fossil fish eye and melanin standards. These results were corroborated by IR data that clearly discriminate between melanin and the two porphyrin-related compounds (Supplementary Figs S10, S11). Hence, we conclude that the microbodies examined herein are indeed the fossilized remains of colour-bearing organelles and that they contain high concentrations of the living organism's original chemistry; that is, components of melanin derived from the pigments of the eye. From our ToF-SIMS analyses of modern retinal melanosomes, it is also clear that other biomolecules, such as proteins and lipids, are essentially gone in the ancient pigment organelles. This suggests that the process of fossilization purifies melanin, whereas more labile organic molecules degrade and disappear. The astonishing stability of melanin, even over deep time, is likely a consequence of its biological role as an energy transducer. Protection against energetic photons (that is, blue and ultra-violet light), as well as quenching of free radicals, are possible because melanin can accommodate both optical and chemical energy and dissipate it as heat, thereby greatly reducing the rate of degradation ${ }^{29,30}$. Because the various physical properties of modern melanins likely are connected to different biological functions ${ }^{31,32}$, our discovery has vast potential to extend the utility 
of molecular structures over deep time, thereby opening a window, based on molecular data, for enhanced understanding of the life and behaviour of animals long extinct.

\section{Methods}

Sample. FUM-N-2050 was collected by one of us (B. P. Schultz) from the Fur Formation of Denmark, without application of preservatives. The fossil was wrapped in aluminium foil and kept isolated within a sealable plastic container until being examined. Fresh aluminium foil was used to cover all work areas, and surgical gloves and sterile scalpels were used in all handling and preparation. Treatment of modern reference samples was identical to that of the fossil structures for all analyses. All experiments were repeated to validate the results.

Scanning electron microscopy. Samples selected for SEM analysis were mounted on glass slides or aluminium blocks using double-sided tape. Initial screening was performed using a Hitachi S-3400N scanning electron microscope on uncoated samples under low vacuum, and the elemental composition was determined via elemental mapping using EDX analysis ( $1900 \mathrm{~s}$ scanning time at $15 \mathrm{kV}, 62.0 \mu \mathrm{A}$ and a working distance of $10 \mathrm{~mm}$ ). Following ToF-SIMS analysis, the samples were sputter-coated with gold and re-examined using a Zeiss Supra 40VP FEG scanning electron microscope ( $2 \mathrm{kV}$, working distance $3-5 \mathrm{~mm}$, Everhart-Thornley secondary electron detector).

Transmission electron microscopy. Microbodies were removed from the eye of FUM-N-2050 using a sterile scalpel and placed in pure alcohol. The alcohol was replaced with acetone, which in turn was stepwise substituted with epoxy resin (AGAR 100 Resin kit, R1031), thereby infiltrating the tissues. The epoxy was left to polymerize at $60^{\circ} \mathrm{C}$ for $48 \mathrm{~h}$. Infiltrated samples were first trimmed with a razor blade and then $2 \mu \mathrm{m}$ thick sections were cut using a glass knife mounted on an ultrotome. Cut sections were stained with Azur-2 methylene blue and examined under a light microscope for a general overview. For the ultra-thin sectioning a diamond knife was used, cutting $50 \mathrm{~nm}$ thick slices that were applied to copper grids. The grids were treated with lead citrate and uranyl acetate for increased contrast and then inserted into a JEOL JEM-1230 transmission electron microscope. The microscope ran at $80 \mathrm{keV}$ and sites of interest were photographed using a MultiScan 701 camera.

Time-of-flight secondary ion mass spectrometry. ToF-SIMS analyses in the static SIMS mode were performed in a ToF-SIMS IV instrument (IONTOF GmbH) using $25 \mathrm{keV} \mathrm{Bi}_{3}{ }^{+}$primary ions and low-energy electron flooding for charge compensation. High mass resolution data were acquired in the bunched mode $(\mathrm{m} / \Delta \mathrm{m}$ $\sim 5000$ ) at a spatial resolution of $\sim 3-4 \mu \mathrm{m}$, whereas high-image resolution data were obtained without bunching $(m / \Delta m \sim 300$, spatial resolution $\sim 0.2-0.5 \mu \mathrm{m})$; in both cases at $256 \times 256$ pixels. Because the positive ion spectra were found to show strong interference with the signal from the sedimentary matrix, only negative ion data are presented herein. The coordinates for all positions investigated were monitored to allow for subsequent SEM analysis of the same areas.

For ToF-SIMS analyses, fossil tissues and sediments were removed using a sterile scalpel and deposited on a substrate of double-sided tape. The synthetic melanin standard was analysed in its powder form, also after deposition on doublesided tape, whereas the Sepia melanin standard was first rinsed in deionized water to remove salts (mainly $\mathrm{NaCl}$ ) and then deposited on a clean silicon wafer and allowed to air dry. The modern retinal melanosomes were deposited on a silicon wafer and air dried before analysis.

ToF-SIMS is a method designed for surface analysis, and it provides mass spectra from the outermost molecular layers $(1-10 \mathrm{~nm})$ of solid sample surfaces. During analysis, a focused beam of high-energy (primary) ions bombards the sample, thereby causing emission of molecules, molecular fragments and atoms (neutral and ionized) from its surface. The ionized fraction of the emitted particles is directed into a time-of-flight mass analyser to produce a mass spectrum. By scanning the primary ion beam over a preselected analysis area on the sample surface, separate mass spectra can be acquired from each raster point within the selected area (typically, $256 \times 256$ raster points and areas ranging in size from $50 \times 50$ to $500 \times 500 \mu \mathrm{m}^{2}$ are examined). The recorded data can be presented in various ways, including: ion images that show the spatial distribution of the signal intensity from selected ions over the analysed area (where pixel brightness reflects the signal intensity); mass spectra from the total analysed area; or mass spectra from selected regions within the analysed area.

Extensive fragmentation of the molecular species on primary ion bombardment was previously a problem; however, modern cluster primary ion sources $\left(\mathrm{Bi}_{\mathrm{n}}{ }^{+}, \mathrm{C}_{60}{ }^{+}\right)$reduce this fragmentation considerably, thereby enabling analysis of molecular species of up to around $2000 \mathrm{Da}$. For larger molecules, such as proteins and polymers, detailed chemical characterization is possible by using characteristic fragment ions, or, preferably (as in this work) patterns of peaks from characteristic fragment ions.

During mass analysis, the primary ions bombard the sample surface in short pulses, and the achievable mass resolution is determined by the shortest possible pulse duration. In contrast, the attainable image resolution is limited by the focus diameter of the primary ion beam. As both the mass resolution and image resolution cannot be at their maxima at the same time (mainly due to space-charge effects), the ToF-SIMS instrument is normally optimized either for maximum mass resolution (bunched mode) or for maximum image resolution (burst alignment mode). In the present contribution, data in the bunched mode were gathered at a mass resolution of $m / \Delta m=5000$ and a lateral resolution of 3-5 $\mu \mathrm{m}$, whereas data in the burst alignment mode were acquired at a mass resolution of around $m / \Delta m=300$ and a lateral resolution down to about $300 \mathrm{~nm}$. However, the lateral resolution also depends on the achievable signal per area unit, which is different for different compounds and monitored ions. Data from the two melanin standards were obtained in the bunched mode to use the high mass resolution for assignment of the peak ions (Table 1). For the fossil sample, the data presented herein were acquired both in the burst alignment mode (Fig. 1; Supplementary Figs S3-S5) and in the bunched mode (Table 1, Fig. 2a; Supplementary Figs S3, S6, S7, and S9). High image resolution was used to allow for reconstruction of mass spectra from small structures, such as individual melanosome-like microbodies in the eye of FUM-N-2050 (these data could then be correlated with microstructures imaged under SEM). The possibility to obtain mass spectra from well-defined, small-sized areas is important when the analyte is localized to specific parts of the surface, thereby minimizing contributions from other compounds that are present at higher concentrations in other regions of the sample surface. However, the sample from the fossil fish eye contained relatively large areas in which the spectra were dominated by melanin, thus allowing for acquisition of data also in the bunched mode (Table 1, Fig. 2a; Supplementary Figs S3, S6, S7, and S9).

ToF-SIMS is generally not used for absolute quantification, owing mainly to matrix effects leading to variations in signal intensities for different chemical environments of the analyte. However, relative quantification; that is, quantification of concentration ratios between different specific compounds, is possible at a semi-quantitative level by using relative sensitivity factors, which can be obtained by analysing standard mixture samples of known composition. To estimate the amount of analyte on the surface from a ToF-SIMS spectrum, it is therefore not the absolute signal intensity (that is, counts) that is primarily relevant but instead the analyte signal intensity relative to the signal intensity from other compounds in the spectrum. In the present work, the fossil fish eye spectra are clearly dominated by peaks characteristic of melanins (based on the spectra from the two melanin standards; Supplementary Fig. S3), whereas peaks that are not present in the melanin standard spectra provide much smaller contributions. Thus, it is possible to conclude that melanin constitutes a major component of the sampled area. Moreover, the low absolute intensities (that is, counts) of the fossil spectra are not a result of low amounts of melanin on the sample surface, but instead reflect the fact that these spectra were reconstructed from very small parts of the analysis areas from which the measurements were taken. Calculation of the number of secondary ions produced per incident primary ion (that is, the secondary ion yield) for selected peaks in the fossil fish eye spectra provides values very similar to those of the melanin standard (Supplementary Table S1); again consistent with high melanin concentrations in these areas.

Compared with other mass spectrometry techniques, such as MALDI-MS/MS or ESI-MS/MS, the main advantage of ToF-SIMS is that it allows for mass spectrometry analysis of solid sample surfaces at sub-micrometre spatial resolution without any pre-treatment of the samples. This means that ToF-SIMS is capable of molecular mapping and mass spectrometry analysis of very small structures $(<1 \mu \mathrm{m})$ embedded in a surrounding matrix; capabilities that were critical when obtaining the data in the present study.

Typically, analysis is done by introducing the entire specimen into the instrument (subject to space limitations, which in our case are $\sim 60 \times 80 \times 6$ (height) $\mathrm{mm}$ ) and navigating to the area of interest using built-in video cameras. In the present case, however, the fossil gave rise to an unacceptably high pressure in the instrument, rendering it necessary to remove small ( $\sim 0.5 \mathrm{~mm}$ in diameter) samples for analysis.

It should also be pointed out that because only the outermost molecular layers of the sample surface are probed during ToF-SIMS analysis, potential contaminations must be carefully considered. Additionally, the choice of proper controls and/or standards is essential for meaningful comparisons. For further practical and methodological information about the use of ToF-SIMS for analysis of geological samples, the reader is referred to Thiel and Sjövall ${ }^{21}$

IR microspectroscopy. Microbodies and other fossil tissues and sediments were removed from FUM-N-2050 using a sterile scalpel, suspended in deionized water, and then placed on sterile $\mathrm{CaF}_{2}$ spectrophotometric windows and left to air dry under a hood at room temperature. Likewise, melanin standard samples were dissolved in deionized water and then casted onto $\mathrm{CaF}_{2}$ spectrophotometric plates. IR microspectroscopy was performed at beamline 73, MAX-IV laboratory, Lund University. The set up combines a Hyperion 3000 microscope and a Bruker IFS66/v FTIR spectrometer. All measurements were recorded in off-line mode using a conventional thermal light source. The microscope was operated in transmission mode with a $170 \times 170 \mu \mathrm{m}$ aperture using a $\times 15$ objective and a condenser along with a single element, $100 \times 100 \mu \mathrm{m}$ MCT detector. This arrangement gives a visible magnification of $\times 215$ for the video camera in the microscope used to locate relevant structures in the samples. 


\section{References}

1. Liu, Y. \& Simon, J. D. Isolation and biophysical studies of natural eumelanins: applications of imaging technologies and ultrafast spectroscopy. Pigment Cell Res. 16, 606-618 (2003).

2. Liu, Y. et al. Comparisons of the structural and chemical properties of melanosomes isolated from retinal pigment epithelium, iris and choroid of newborn and mature bovine eyes. Photochem. Photobiol. 81, 510-516 (2005)

3. Gunderson, A. R., Frame, A. M., Swaddle, J. P. \& Forsyth, M. H. Resistance of melanized feathers to bacterial degradation: is it really so black and white? J. Avian Biol. 39, 539-545 (2008).

4. Raposo, G. \& Marks, M. S. Melanosomes - dark organelles enlighten endosomal membrane transport. Nat. Rev. Mol. Cell Biol. 8, 786-797 (2007).

5. Wuttke, M. 'Weichteil-Erhaltung' durch lithifizierte Mikroorganismen bei mittel-eozänen Vertebraten aus den Ölschiefern der 'Grube Messel' bei Darmstadt. Senck. Leth. 64, 509-527 (1983).

6. Davis, P. G. \& Briggs, D. E. G. Fossilization of feathers. Geology 23, 783-786 (1995).

7. Gupta, N. S. et al. Molecular taphonomy of macrofossils from the Cretaceous Las Hoyas Formation, Spain. Cret. Res. 29, 1-8 (2008).

8. Vinther, J., Briggs, D. E. G., Prum, R. O. \& Saranathan, V. The colour of fossil feathers. Biol. Lett. 4, 522-525 (2008).

9. Vinther, J., Briggs, D. E. G., Clarke, J., Mayr, G. \& Prum, R. O. Structural coloration in a fossil feather. Biol. Lett. 6, 128-131 (2010).

10. Zhang, F. et al. Fossilized melanosomes and the colour of Cretaceous dinosaurs and birds. Nature 463, 1075-1078 (2010).

11. Li, Q. et al. Plumage color patterns of an extinct dinosaur. Science 327, 1369-1372 (2010).

12. Reitner, J. In Encyclopedia of Geobiology (eds Reitner, J., Thiel, V.) 136-137 (Springer, 2011)

13. Knight, T. K., Bingham, P. S., Lewis, R. D. \& Savrda, C. E. Feathers of the Ingersoll Shale, Eutaw Formation (Upper Cretaceous), eastern Alabama: the largest collection of feathers from North American Mesozoic rocks. Palaios 26, 364-376 (2011).

14. Barden, H. E. et al. Morphological and geochemical evidence of eumelanin preservation in the feathers of the Early Cretaceous bird, Gansus yumenensis. PLoS ONE 6, e25494 (2011).

15. Wogelius, R. A. et al. Trace metals as biomarkers for eumelanin pigment in the fossil record. Science 333, 1622-1626 (2011).

16. Carney, R. M., Vinther, J., Shawkey, M. D., D’Alba, L. \& Ackermann, J. New evidence on the colour and nature of the isolated Archaeopteryx feather. Nat. Commun. 3, 637 (2012).

17. Li, Q. et al. Reconstruction of Microraptor and the evolution of iridescent plumage. Science 335, 1215-1219 (2012).

18. Lindgren, J., Caldwell, M. W., Konishi, T. \& Chiappe, L. M. Convergent evolution in aquatic tetrapods: insights from an exceptional fossil mosasaur PLoS ONE 5, e11998 (2010).

19. Hitchcock, A. P. et al. Soft X-ray spectromicroscopy of nickel sorption in a natural river biofilm. Geobiology 7, 432-453 (2009).

20. Schweitzer, M. H. Soft tissue preservation in terrestrial Mesozoic vertebrates Annu. Rev. Earth Planet. Sci. 39, 187-216 (2011).

21. Thiel, V. \& Sjövall, P. Using time-of-flight secondary ion mass spectrometry to study biomarkers. Annu. Rev. Earth Planet. Sci. 39, 125-156 (2011).

22. Willumsen, P. S. Palynology of the Lower Eocene deposits of northwest Jutland, Denmark. Bull. Geol. Soc. Denmark 52, 141-157 (2004).

23. Westerhold, T., Röhl, U., McCarren, H. K. \& Zachos, J. C. Latest on the absolute age of the Paleocene-Eocene thermal maximum (PETM): new insights from exact stratigraphic position of key ash layers +19 and -17 . Earth Planet. Sci. Lett. 287, 412-419 (2009).

24. Meredith, P. \& Sarna, T. The physical and chemical properties of eumelanin Pigment Cell Res. 19, 572-594 (2006).
25. Perna, G., Palazzo, G., Mallardi, A. \& Capozzi, V. Fluorescence properties of natural eumelanin biopolymer. J. Luminesc. 131, 1584-1588 (2011)

26. Plonka, P. M. \& Grabacka, M. Melanin synthesis in microorganismsbiotechnological and medical aspects. Acta Biochim. Polon. 53, 429-443 (2006).

27. Bauder, C., Ocampo, R., Callot, H. J. \& Albrecht, P. Structural evidence for heme fossils in Messel Oil Shale (FRG). Naturwissenschaften 77, 378-379 (1990).

28. Ping'an, P., Eglinton, G., Jiamo, F. \& Guoying, S. Biological markers in Chinese ancient sediments. 1. Geoporphyrins. Energy Fuels 6, 215-225 (1992).

29. Meredith, P. \& Riesz, J. Radiative relaxation quantum yields for synthetic eumelanin. Photochem. Photobiol. 79, 211-216 (2004).

30. McGraw, K. J. The antioxidant function of many animal pigments: are there consistent health benefits of sexually selected colourants? Anim. Behav. 69 , 757-764 (2005)

31. Ortonne, J.- P. Photoprotective properties of skin melanin. Br. J. Dermatol. 146, 7-10 (2002).

32. Simon, J. D., Hong, L. \& Peles, D. N. Insights into melanosomes and melanin from some interesting spatial and temporal properties. J. Phys. Chem B 112, 13201-13217 (2008).

33. Watt, A. A. R., Bothma, J. P. \& Meredith, P. The supramolecular structure of melanin. Soft Matter 5, 3754-3760 (2009).

34. Chiba, K., Akamatsu, T. \& Kawamura, M. Intensity variation dependence of secondary ion emission of carbon clusters from graphite, diamond-like carbon and diamond surfaces on number of carbon atoms. Chem. Phys. Lett. 419, 506-510 (2006).

35. Bosch-Reig, F., Gimeno-Adelantado, J. V. \& Moya-Moreno, M. C. M. FTIR quantitative analysis of calcium carbonate (calcite) and silica (quartz) mixtures using the constant ratio method. Application to geological samples. Talanta 58, 811-821 (2002).

36. Kaxiras, E., Tsolakidis, A., Zonios, G. \& Meng, S. Structural model of eumelanin. Phys. Rev. Lett. 97, 218102-1-218102-4 (2006)

\section{Acknowledgements}

R. Wallén, C. Alwmark, C. Rassmusen, P. Ekström, C. Heim and T. Leefmann assisted during the laboratory work. This research was supported by grants from the Swedish Research Council (J.L., P.U., D.E.N.), Knut and Alice Wallenberg foundation (P.U.), VINNOVA Swedish Governmental Agency for Innovation Systems (P.S.), the German Research Foundation (DFG Research Unit 571, V.T.) and the Royal Physiographic Society in Lund (J.L.).

\section{Author contributions}

J.L., P.U., P.S. and D.E.N. designed the project, planned the experiments and performed the data analyses. J.L. wrote the manuscript. P.S. performed the ToF-SIMS analyses and A.E. conducted the IR experiments. P.S. and J.L. performed the SEM analyses. B.P.S. and V.T. participated in the data analyses and provided fossils and microbial mat samples. All authors discussed the results and provided input on the manuscript.

\section{Additional information}

Supplementary Information accompanies this paper at http://www.nature.com/ naturecommunications

Competing financial interests: The authors declare no competing financial interests.

Reprints and permission information is available online at http://npg.nature.com/ reprintsandpermissions/

How to cite this article: Lindgren, J., et al. Molecular preservation of the pigment melanin in fossil melanosomes. Nat. Commun. 3:824 doi: 10.1038/ncomms1819 (2012). 